\title{
Physical parameters of pre-main sequence stars in open clusters ${ }^{\star}$
}

\author{
A. J. Delgado ${ }^{1}$, E. J. Alfaro ${ }^{1}$, and J. L. Yun ${ }^{2}$ \\ ${ }^{1}$ Instituto de Astrofísica de Andalucía, CSIC, Apdo 3004, 18080-Granada, Spain \\ e-mail: [delgado; emilio]@iaa.es \\ 2 Centro de Astronomia e Astrofísica da Universidade de Lisboa, Observatório Astronómico de Lisboa, Tapada da Ajuda, \\ 1349-018 Lisboa, Portugal \\ e-mail: joao.yun@oal.ul.pt
}

Received 10 January 2011 / Accepted 17 May 2011

\begin{abstract}
Aims. The aims are twofold: we search and analyse pre-main sequence (PMS) stars in Galactic young open clusters (YOCs) to determine the ages, masses, and spatial distribution of PMS members in YOCs, and to check and compare the performances of different model isochrones.

Methods. We compare UBVRI photometric observations to theoretical isochrones in the photometric diagrams. The comparison simultaneously provides membership assignments for both main sequence (MS) and PMS stars and estimates for the physical properties of the cluster candidate members: masses, ages, and spatial distribution inside the cluster.

Results. The photometric measurement of an average $(U-V)$ excess, supposedly indicative of accretion disks, is considered prior to membership assignment. This photometric excess is correlated with cluster age, suggesting that disks able to show up in $(U-V)$ excess vanish at ages around $5 \mathrm{Myr}$. We find that comparing photometry to models in the colour-magnitude (CM) diagram leads to better results for the masses than performing the comparison in the theoretical HR plane. The obtained cluster mass functions show a marginal steepening with cluster age. Significant variations in the mass function slopes are found with the models used in member selection. The clusters NGC 3293 and NGC 2362 are found to have mass functions flatter than the Salpeter slope for all models considered. The relation between the calculated dispersion of PMS age and the characteristic clustering scale of the cluster shows interesting agreement with previous findings in star-forming regions in a wide range of scales. Finally, the ratio of characteristic clustering scales for PMS candidate members in different mass ranges can be interpreted as suggesting mass segregation, in the sense of a relatively wider spatial distribution for the lower mass members in older clusters.

Conclusions. The relations between the different cluster parameters show that the procedure applied to assign cluster membership and to measure physical parameters for the selected members is well founded.
\end{abstract}

Key words. stars: formation - stars: pre-main sequence - open clusters and associations: general

\section{Introduction}

The primary aim of a general investigation of star formation is to obtain reliable measurements of the distribution of the forming objects in mass, age, and spatial structure. A simultaneous aim of the investigation is to constrain the models used in determining these physical parameters.

Further objectives may include details of the time evolution of these properties and their dependence on other parameters, such as the chemical composition, the total mass of the initial cloud, or the environmental conditions. But the fundamental aims are to tune the theoretical models and to obtain accurate values of masses and ages.

In this context, stellar clusters are the most reliable tool for measuring the distances, masses, and ages of stars. Young open clusters (YOCs), in particular, serve to deepen our understanding of stars in early evolutionary phases, including the premain sequence (PMS) evolution. Our work deals with YOCs of ages around $10 \mathrm{Myr}$, which offer some clear advantages to the study of the star formation process in clusters. These objects are

* The photometric catalogue is available in electronic form at the CDS via anonymous ftp to cdsarc.u-strasbg. fr (130.79.128.5) or via

http://cdsweb.u-strasbg.fr/cgi-viz/qcat?]/A+A/531/A141 usually not embedded in the remnants of the dust and gas clouds where they formed, and can be studied with multiwavelength photometric observations, thereby covering the optical and the infrared ranges. For these clusters, reliable determinations of distance and absorption are possible, which greatly help in further determinations of physical parameters of the PMS cluster members, such as mass and age.

In recent years, detailed new observations of some particular YOCs have enlarged the data available to test and constrain model predictions. Primarily, X-ray detections and $\mathrm{H} \alpha$ emission, together with spectroscopically determined spectral types, provide assessment of cluster members, and, subsequently, models are used to obtain masses and ages (Flaccomio et al. 2006, F06 in the following; Dahm et al. 2007, D07 in the following). In this context, several sets of PMS isochrone models have been published, which cover different ranges in star mass and other physical parameters (see Hillenbrand \& White 2004, H04 in the following).

Recent studies have compared models to observations on the basis of synthesized clusters, which were simulated from different models and which have assumed contributions from the expected sources of uncertainty (Hillenbrand et al. 2008). Another approach to assessing age determinations of theoretical models has been advanced recently, and is based on the analysis of 
stellar pulsation of PMS stars and comparison with predictions of model interiors (Zwintz et al. 2008).

The measurement of physical parameters such as mass and age is achieved through comparing observations with evolutionary stellar interior models in the HR diagram. This comparison between models and observations can be performed in two ways. The first consists of calculating luminosity and effective temperature from observed colours, and afterwards the physical parameters are read from the theoretical isochrones in the HR diagram (examples for NGC 2264 by Rebull et al. 2002, R02 in the following; F06). This approach is used in the methods for age measurement reviewed by Naylor et al. (2009). This procedure has the advantage of more accurately considering extinction for the individual stars. But the comparison to models needs additional information, such as spectral types and, most important, membership confirmation, which is usually lacking. The second approach consists of translating the theoretical isochrones to colours and absolute visual magnitudes and of comparing to observations in the photometric diagrams (D07, also for NGC 2264). For a general sample of clusters, the conversion from theoretical luminosity and effective temperature to photometric colours is preferred, in particular if the extinction does not show values or degrees of variability that are too high. The transformed isochrones can then be used as reference lines to measure colour excess and distance. Interestingly, this approach allows simultaneous study of three questions: a) assignment of cluster membership; b) determination of the physical parameters for the candidate members; and c) test of the performances of different evolutionary calculations.

In this paper we present the results of the methodology used to obtain basic physical information on the PMS member stars in YOCs. It follows the second approach described in the previous paragraph. The clusters have been selected in an estimated age range between 5 and $30 \mathrm{Myr}$ and affected by small reddening. They were chosen for their location both at relatively close Sun distances and in a narrow range of Galactic longitude. With these criteria we expect to minimize the influence of the factors that introduce uncertainty into the determination of cluster parameters. The selected clusters can be observed well with smallto-medium size telescopes, mostly in a single campaign, to make the photometry as homogeneous as possible. With the mentioned requirements, we expect the detection of PMS cluster members down to at least early $\mathrm{K}$ spectral types.

The use of $U B V R I$ photometry, adequately compared to theoretical isochrones, provides homogeneous samples of candidate members, at the same time giving a measurement of their ages and masses. This can be used to investigate basic features of the star-forming process, as described by the spatial distributions of the PMS members inside the cluster, the mass function, and the age structure. Beyond the obtaining of specific relations between parameters, our main aim is to show the ability of our procedure to advance in the desired direction: defining PMS member samples in young clusters across the whole Galactic disk and opening the possibility of an improved and systematic checking of PMS evolutionary models.

In Sect. 2 we review the procedure applied to estimate PMS membership through isochrone fitting, and describe the comparisons between the members selected using our method and published results on some YOCs. In Sect. 3, the results of our method, applied to 11 southern YOCs in the age range 5 to $30 \mathrm{Myr}$, are presented. In Sect. 4, the main results and conclusions are outlined.
Table 1. Clusters observed.

\begin{tabular}{lrrrrcr}
\hline \hline $\begin{array}{l}\text { Cluster } \\
\text { name }\end{array}$ & $\begin{array}{r}l \\
\text { deg }\end{array}$ & $\begin{array}{r}b \\
\text { deg }\end{array}$ & $E(B-V)$ & $D M$ & $\begin{array}{c}\text { log Age } \\
\text { yr }\end{array}$ & $N$ \\
\hline NGC 2362 & 235.65 & -3.84 & 0.12 & 10.8 & $6.5 \pm 0.08$ & 6 \\
NGC 2367 & 238.18 & -5.55 & 0.38 & 12.3 & $6.6 \pm 0.13$ & 5 \\
NGC 3293 & 285.85 & 0.07 & 0.29 & 12.0 & $6.8 \pm 0.07$ & 31 \\
Collinder 228 & 287.52 & -1.03 & 0.32 & 12.0 & $6.7 \pm 0.10$ & 4 \\
Hogg 10 & 290.80 & 0.10 & 0.36 & 11.9 & $7.0 \pm 0.18$ & 5 \\
Hogg 11 & 290.89 & 0.14 & 0.30 & 11.9 & $6.8 \pm 0.02$ & 4 \\
Trumpler 18 & 290.99 & -0.14 & 0.30 & 10.6 & $7.5 \pm 0.29$ & 3 \\
NGC 3590 & 291.21 & -0.18 & 0.52 & 11.7 & $7.2 \pm 0.07$ & 9 \\
NGC 4103 & 297.57 & 1.18 & 0.32 & 11.5 & $7.3 \pm 0.06$ & 11 \\
NGC 4463 & 300.65 & -2.01 & 0.39 & 11.1 & $7.3 \pm 0.22$ & 5 \\
NGC 5606 & 314.84 & 0.99 & 0.49 & 11.8 & $7.0 \pm 0.11$ & 7 \\
\hline
\end{tabular}

Notes. Column 1: cluster name; 2: galactic longitude; 3: galactic latitude; 4: colour excess; 5: distance modulus; 6: logarithmus of age (yr); $7:$ number of stars used in the age calculation.

\section{Membership determination}

In the present study we focus on the results for 11 southern YOCs, included in our previous papers (Delgado et al. 2006; Delgado et al. 2007. DAY07 in the following). The clusters selected are listed in Table 1, together with their Galactic coordinates ( $l, b$ in degrees), colour excesses, distance moduli, and ages with uncertainty and indication of the number of stars used in the age calculation.

The comparison with the zero-age main sequence (ZAMS) and to Padova isochrones by Girardi et al. (2002) in the colourmagnitude (CM) diagrams provides mass estimates for the MS cluster members. The evolved MS members (designed in the following as postMS) are used to calculate the cluster ages and uncertainties listed in Table 1 (DAY07). Values for the distance, colour excess, and ages of Hogg 10 and Hogg 11 have been recalculated in this work. The uncertainty of the age values, given by the standard error of the mean in each case, has typical values between 0.1 and 0.3 in the logarithm, but the age values themselves depend on the model isochrones used.

\subsection{UV excess of PMS cluster members}

Prior to determining the membership, we analyse the possible presence of ultraviolet excess in the candidate members. In R02 it is suggested that several stars among the PMS cluster members in NGC 2264 exhibit excess continuum emission in ultraviolet wavelenghts, which should be originating in accretion disks. This is actually one of the disk signatures they explore, together with near infrared (NIR) excess and $\mathrm{H} \alpha$ emission. They claim that the excess is present in the $U$ band, and absent from $R$ and $I$ bands. With their own spectral classification, they use the spectral-type related intrinsic colours to estimate the amount of ultraviolet excess, as observable in the $(U-V)$ colour index when plotted against $(R-I)$ in the corresponding colour-colour (CC) diagram. The so-called $U V$ excess is defined as the difference between the dereddened $(U-V)$ and the intrinsic $(U-V)$, obtained from the spectroscopically derived spectral type.

We reproduce these results on the basis of photometry, an approach discussed by Rebull et al. (2000) and R02. It is based on the assumption that the presence of an accretion disk does not produce any excess in the $R$ and $I$ bands, so that the red colour indices $(R-I)$ and even $(V-I)$ are free of this effect. Here we assume the median colour excess $E(B-V)$ for calculated 
A. J. Delgado et al.: Physical parameters of PMS stars in YOCs

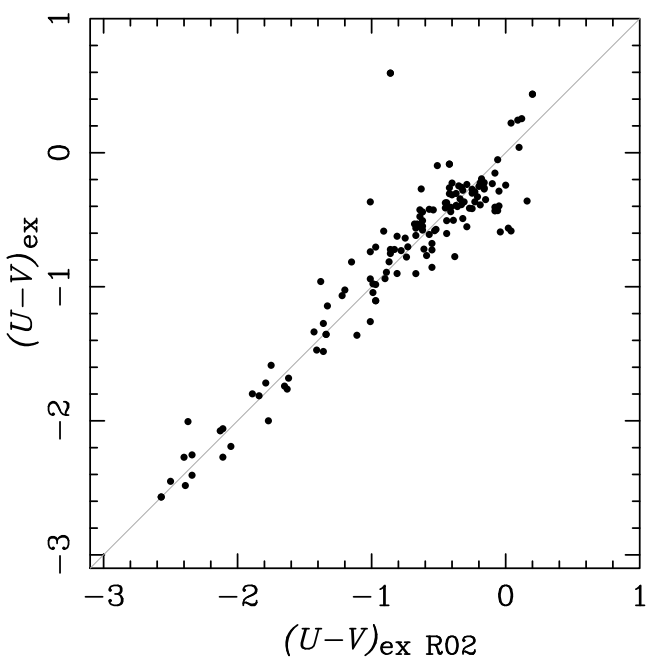

Fig. 1. Excesses in $(U-V)$ for PMS members in NGC 2264 calculated from photometry (see text), plotted versus spectroscopic $(U-V)$ excess by R02. The continuous line shows the slope 1 .

MS members (see DAY07), together with the extinction law by Fitzpatrick (1999) to provide a colour excess $E(R-I)$ and the corresponding intrinsic colour $(R-I)_{0}$. This intrinsic colour is calculated for every star, and provides a spectral type estimator, which can be used in the same way as in R02 to calculate the $(U-V)$ excess.

In Fig. 1 we show the results of this calculation for cluster PMS members, plotted against the values listed by R02. The plot shows a good performance of the assumption, which allows us to estimate $U V$ excesses in this way for any observed cluster, for which only photometric measurements are obtained, and membership and spectral type information is lacking. Excesses in $(U-B)$ and $(B-V)$ are calculated with the relations $(U-B)_{\mathrm{ex}}=$ $0.76 \times(U-V)_{\mathrm{ex}},(B-V)_{\mathrm{ex}}=0.24 \times(U-V)_{\mathrm{ex}}$, obtained from linear correlations to the calculated $(U-V)$ excesses in NGC 2264. We follow the considerations by Rebull et al. (2000), and apply only those excesses which correspond to $(U-V)_{\mathrm{ex}}<-0.4$.

The influence of the $U V$ excess is only appreciable for the youngest clusters in our sample. In Fig. 2 we show a plot of the median $U V$ excesses against cluster age. We include values for the clusters NGC 2264 (2.5 Myr given by D07) and NGC 1893 (4 Myr, adopted by Sharma et al. 2007). The error bar in the $U V$ excess reproduces the standard error of the mean of the differences between photometric and spectroscopic values in NGC 2264.

A trend with age can be surmised in Fig. 2, which suggests an age limit of around $5 \mathrm{Myr}$ for the presence of disks, capable of producing $U V$ excess. We recall in this context results obtained previously, with time scales for disk dissipation from 4 to $6 \mathrm{Myr}$ (Haisch et al. 2001; Sicilia-Aguilar et al. 2005).

\subsection{Fitting to model isochrones}

The procedure for selecting PMS cluster members has been explained in detail in DAY07, and we summarize it here. The measurement of colour excess and distance for main sequence (MS) cluster members is carried out by ZAMS fitting. These values of reddening and distance, estimated from all selected MS candidate members, are then used as reference values to assign membership to all measured stars. The theoretical PMS isochrones converted to the photometric diagrams are used in

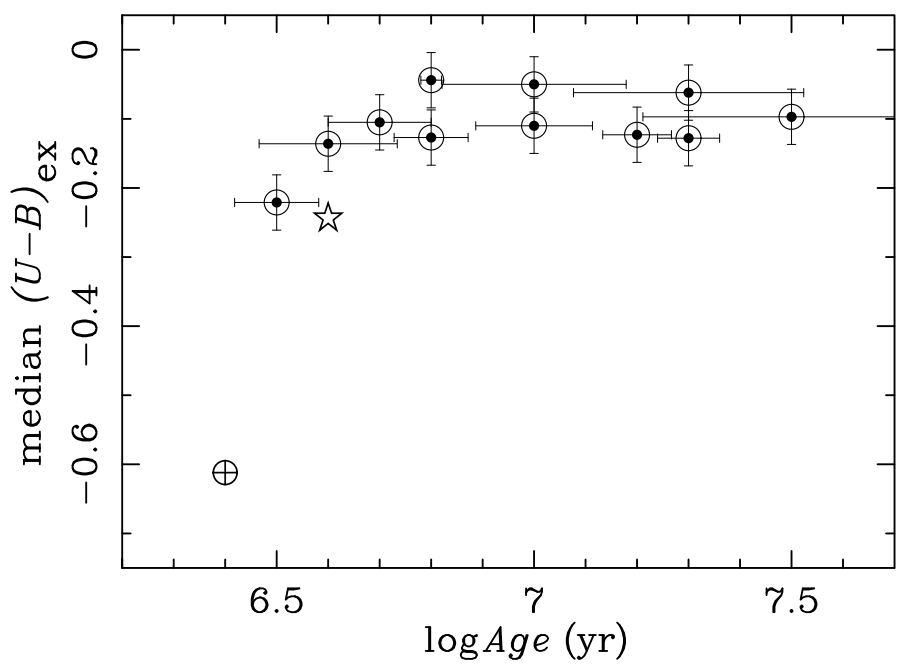

Fig. 2. Median $(U-B)$ excess for the stars with negative calculated value (see text), plotted versus cluster age, obtained from quantitative comparison to Padova isochrones. The plotted error bars reproduce the standard error of the mean in each case. Values for NGC 2264 (crossed circle) and NGC 1893 (star) are also plotted for comparison.

this assignment as reference lines to measure colour excess and distance. Membership is established when the measured colour excesses and distance coincide, within the errors, with the reference values and any particular star can be assigned as a PMS member from the comparison to PMS isochrones in several CM diagrams. Furthermore, the isochrones that provide membership assignment also provide a mass and age value for the candidate members, calculated as averages of the values extracted from all assignments.

We introduce here some improvements in the membership determination with respect to DAY07. First, the estimate of reddening and membership for those stars without valid $U$ measurement considers the colour excess values calculated for all stars with measurement in all five $U B V R I$ colours and with a membership assignment. Second, we consider the possible presence of ultraviolet excess in the stars' colours, as explained in Sect. 2.1. As seen, this factor turns out to be unimportant for cluster ages above 6 Myr. Third, in relation to DAY07, the use of Yale PMS isochrones (Yi et al. 2001) is added. The four models considered are by D'Antona \& Mazzitelli (1997), Palla \& Stahler (1999), Siess et al. (2000) and Yi et al. (2001), referred to in the following as D97, P99, S00, and Y01, respectively. All of them were converted to the CM diagrams with the relations from Kenyon \& Hartmann (1995).

A catalogue with the photometric results for the 27040 stars in the 11 cluster fields is contained in a catalogue available at the CDS and from http://ssg.iaa.es. It supersedes the members list published in DAY07. The catalogue contains the following information: the cluster identification in the OCL catalogue in Col. 1; the 2MASS identification (2) and our own identification (3); right ascension (4) and declination (5) for the Epoch 2000; colour indices with errors, $V, \sigma(V),(U-B), \sigma(U-$ $B),(B-V), \sigma(B-V),(V-R), \sigma(V-R)$, and $(V-I), \sigma(V-I)(6$ to 15$)$; type of membership, and the values of age, mass, luminosity, and effective temperature calculated with the PMS models of D97 (16-20); type of membership, and the values of age, mass, luminosity, and effective temperature calculated with the PMS models of P99 (21-25); type of membership, and the values of age, mass, luminosity, and effective temperature calculated 
with the PMS models of S00 (26-30); type of membership, and the values of age, mass, luminosity, and effective temperature calculated with the PMS models of Y01 (31-35).

The procedure for membership assignment in principle has two main shortcomings. First, the required conversion from luminosity and effective temperature to intrinsic colours and bolometric corrections is not necessarily the same as the one used for MS stars. This difficulty is also present when converting observed colours to theoretical quantities. The differences are, however, of remarkable importance for PMS stars of class II and younger (Delgado et al. 1998). For class III objects, the assumption of the validity of the calibration used for MS stars is a reasonable approximation (Kenyon \& Hartmann 1995).

Second, the estimate of absorption for each individual star is necessary to calculate distance. For many stars in the cluster field, and in particular the faintest ones, this estimate is probably affected by undetected variations, and might lead to inaccurate estimates both for the membership, and for the mass and age of the candidate members. It is important to point out that this source of uncertainty is unavoidable for clusters where information about spectral type is absent. This is indeed the general case. Follow-up spectroscopic observations are therefore important, both for assessing the membership estimates and simultaneously determining the actual differences between the performances of the different models.

Two more remarks need to be made here. First, in some clusters the procedure for selecting MS members (DAY07) assigns MS membership to an appreciable number of stars that are fainter than the brightest PMS selected members. These are discussed by Delgado et al. (2010) in the case of another cluster of interest, Dolidze 25, located inside a larger star-forming region Sh 2-284. In this context we mention the recent work by Beccari et al. (2010), where progressive star formation in the young galactic super star cluster NGC 3603 is proposed, with one third of the PMS members found to be older than $10 \mathrm{Myr}$, while the ages of the remaining members range from 1 to 10 Myr. For the clusters in our sample, further spectroscopic assessment is needed to confirm that these stars are actually cluster members. Their MS membership is included in the final catalogue, but they are not considered in the analysis of the results in the present paper. Second, as mentioned, the PMS membership assignment can be obtained with respect to isochrones in 2,3 , or 4 CM diagrams. Unless otherwise stated, in the following discussions we use the term "PMS member" to name a star with uncertainties in all photometric indices smaller than 0.05 , and with membership assignment in at least 3 CM diagrams (3CM-PMS membership assignment).

\subsection{Comparison to published results}

Previous studies contain lists of X-Ray sources in the cluster NGC 2362 (Delgado et al. 2006; Damiani et al. 2006) and stars with $\mathrm{H} \alpha$ emission or Li $6707 \AA$ absorption (Dahm 2005). Stars with any of these features that are located in the area covered by the isochrones in the CM diagrams are considered cluster members. We name them $P$-members. Eighty five percent of these $P$-members are selected in our procedure, using the S00 models. On the other hand, $70 \%$ of our PMS members with S00 models are also $P$-members. This gives a coincidence (agreement of assignments in both directions) of $60 \%$. This percentage decreases to around $40 \%$ with P99 and Y01 models.

In this context, a relatively higher degree of agreement is found for PMS candidates of later spectral types. A similar result is found for NGC 1893, another young cluster located relatively farther in the direction of the Galactic anticentre. We also tested our method for this cluster using the $U B V R I$ photometry by Sharma et al (2007). We consider as PMS $P$-members all stars in the Sharma et al. photometry that are also classified as class II and III sources by Caramazza et al. (2008). Our method of membership assignment with S00 models assigns PMS membership for $74 \%$ of these $P$-members. This percentage turns out to be higher for stars fainter than $V=17(76 \%)$ than for stars brighter than this $(71 \%)$. This suggests that PMS stars of earlier types, around AF, might be more difficult to detect in X rays than PMS stars of GK types (Damiani et al. 2006).

Finally, we used the published data on NGC 2264 as a template to analyse the outcome of our procedure. Its age is close to 3 Myr (Sung 2004, D07), and it is known to host a wellpopulated sequence of PMS stars, ranging from spectral types AF down to the less massive T Tauri stars. Since the first study by Walker (1956), numerous works have been devoted to this cluster (Dahm 2008 contains a complete list of references). $U B V R I$ photometry has been published by several authors. We use here the results by R02 and the UBV photometry by Walker (1956) for stars with $V \leq 14$. The results on PMS membership, mass, and age by R02, and also by F06 and D07 are considered. The cross-identifications between different studies and the basic photometric information were taken from WEBDA (http:// www. univie.ac.at/webda/). For the distance of NGC 2264 we adopt the value given by Baxter et al. (2009).

The application of our procedure to the combined photometric results from Walker (1956) and R02 provides 2CM-PMS membership assignment for the $91 \%$ of the joint member samples by R02, F06 and D07. The percentage decreases to $63 \%$ when considering $3 \mathrm{CM}-\mathrm{PMS}$ membership. The reason for this is probably the highly variable absorption in the cluster from MS to PMS members, which makes the colour excess estimate for PMS candidates uncertain on the sole basis of photometry. In this comparison we used S00 isochrones for metallicity $Z=0.02$, which are those used by R02 to obtain their mass values.

The physical parameters of the PMS member samples in NGC 1893 and NGC 2264 mentioned above, as well as the MS members obtained from the same photometric studies cited (Sharma et al. 2007; Walker 1956; R02), will be used as examples in the rest of the paper.

\section{Physical parameters of PMS candidate members}

We now turn to a discussion of the results obtained for the physical parameters of the assigned member samples. The general results for the clusters are listed in Table 2. All the results in this table for which the use of models is involved are obtained with S00 models.

\subsection{Masses of PMS members}

As explained before (see DAY07, and Sect. 2 above), the membership assignment to a particular star can happen with respect to several isochrones. This provides several age and mass values for the particular candidate, and the averages and rms deviations of all these values, are taken as the mass and age values, with uncertainties, of the particular PMS candidate member. How do these values compare to previous results? In this section we refer to previous results on PMS masses, and discuss the differences between the values obtained using various evolutionary models. 
Table 2. Physical parameters of the observed clusters.

\begin{tabular}{|c|c|c|c|c|c|c|c|c|c|c|}
\hline $\begin{array}{l}\text { Cluster } \\
\text { name }\end{array}$ & $(U-B)_{\mathrm{ex}}$ & $N_{\mathrm{MS}}$ & $N_{\mathrm{PMS}}$ & MF & $\mathrm{MF}_{\mathrm{PMS}}$ & $\begin{array}{c}\text { Age }_{\text {PMS }} \\
\text { Myr }\end{array}$ & $\underset{\mathrm{Myr}}{\Sigma}$ & $\begin{array}{c}\Delta \\
\mathrm{pc}\end{array}$ & $\begin{array}{c}\Delta_{\text {Low } M} \\
\text { pc }\end{array}$ & $\begin{array}{c}\Delta_{\mathrm{High} M} \\
\mathrm{pc}\end{array}$ \\
\hline NGC 2362 & -0.221 & 28 & 89 & $-0.74 \pm 0.21$ & $-2.30 \pm 0.31$ & 5.44 & 1.95 & 1.82 & 1.78 & 1.86 \\
\hline NGC 2367 & -0.136 & 11 & 132 & $-1.13 \pm 0.29$ & $-1.95 \pm 1.12$ & 5.48 & 2.57 & 4.26 & 4.37 & 4.07 \\
\hline NGC 3293 & -0.127 & 88 & 231 & $-0.64 \pm 0.19$ & $-1.63 \pm 0.42$ & 6.36 & 2.45 & 3.53 & 3.77 & 3.32 \\
\hline Collinder 228 & 0.105 & 16 & 305 & $-1.93 \pm 0.25$ & $-3.21 \pm 0.27$ & 6.46 & 2.53 & 3.60 & 3.52 & 3.68 \\
\hline Hogg 10 & -0.050 & 20 & 270 & $-1.33 \pm 0.47$ & $-3.35 \pm 0.51$ & 6.31 & 2.47 & 3.25 & 3.26 & 3.08 \\
\hline Hogg 11 & 0.044 & 13 & 393 & $-1.20 \pm 0.43$ & $-3.88 \pm 0.72$ & 6.41 & 2.42 & 3.96 & 3.93 & 3.90 \\
\hline Trumpler 18 & -0.097 & 16 & 126 & $-1.07 \pm 0.29$ & $-2.09 \pm 0.58$ & 4.59 & 2.84 & 2.01 & 2.04 & 2.18 \\
\hline NGC 3590 & -0.123 & 37 & 406 & $-1.51 \pm 0.34$ & $-2.29 \pm 0.35$ & 6.28 & 2.43 & 2.98 & 2.98 & 2.94 \\
\hline NGC 4103 & 0.128 & 49 & 214 & $-1.31 \pm 0.27$ & $-2.41 \pm 0.56$ & 6.18 & 2.33 & 2.92 & 2.97 & 2.78 \\
\hline NGC 4463 & -0.062 & 21 & 151 & $-1.35 \pm 0.25$ & $-3.75 \pm 0.68$ & 6.23 & 2.64 & 2.43 & 2.49 & 2.23 \\
\hline NGC 5606 & -0.110 & 21 & 215 & $-1.53 \pm 0.31$ & $-3.40 \pm 0.63$ & 6.03 & 2.77 & 3.27 & 3.21 & 3.32 \\
\hline
\end{tabular}

Notes. Column 1: cluster name; 2: $(U-B)$ excess (Sect. 2.1); 3: number of MS+postMS members; 4: number of PMS members (Sect. 2.2); 5: slope of the mass functions, $\delta \log N_{M} / \delta \log M$, for all the members of mass above the approximate completeness limit (Sect. 3.1.2); 6: the same slope for the mass function calculated with only the PMS members; 7, 8: average age of the PMS members (7), and its rms deviation (8); 9: average value of the distances between all pairs of PMS members; 10, 11: this same quantity calculated for the distribution of PMS members of low mass $\left(1-2 M_{\odot}\right)(10)$ and high mass $\left(2-3.5 M_{\odot}\right)(11)$.

\subsubsection{Masses of PMS members in NGC 2264}

To illustrate the results of our method of determining membership and the physical parameters of members, we discuss the results on the masses of PMS members in NGC 2264 in this section. For this cluster the comparison to isochrones can be performed on the basis of a previously established member population by independent means, and it therefore offers a good possibility of testing the reliability of our procedure.

We extracted two sets of mass values from each of the published works (R02, F06, D07). First, we have the three sets of published values, all of them obtained with S00 models, which we call $P$-values in the following. These three sets of $P$-values differ from each other. Values from F06 and D07 do not differ systematically, although the dispersion of the difference is high $\left(M_{\mathrm{D} 07}-M_{\mathrm{F} 06}=0.005 \pm 0.26\right)$. The R02 values are systematically lower than both the F06 and D07 values $\left(M_{\mathrm{R} 02}-M_{\mathrm{F} 06}=\right.$ $\left.-0.14 \pm 0.26, M_{\mathrm{R} 02}-M_{\mathrm{D} 07}=-0.22 \pm 0.28\right)$. A second set of mass values was obtained by shifting the transformed model isochrones in the CM $V,(B-V)$ diagram, according to the published values of distance and absorption for every individual star, and reading its mass from the isochrone at nearest distance. The mass values from this second procedure are named $T$-values. The $P$ - and $T$-values should be similar to each other, since they were obtained from the same observed colours, and were compared to the same isochrone models. However, they differ significantly.

The results attained by $\mathrm{H} 04$ on star masses from different evolutionary models show that the masses of PMS stars predicted by all models are systematically lower than dynamical masses. In Fig. 3 we reproduce the comparative plots produced by H04 in their Figs. 4 and 5. Here we plot the difference of $T$ - minus $P$-values, compared with $P$-values. The plot shows that $T$-values are systematically higher than the $P$-values. On the other hand, the results by H04 show that the values from the models (associated to our $P$-values here) are less than "correct" dynamical masses. The differences shown in our plots and in those by $\mathrm{H} 04$ are furthermore of the same order. This means that the $T$-procedure produces higher mass values than the published $P$-values, so mass results in better agreement with the dynamical values.

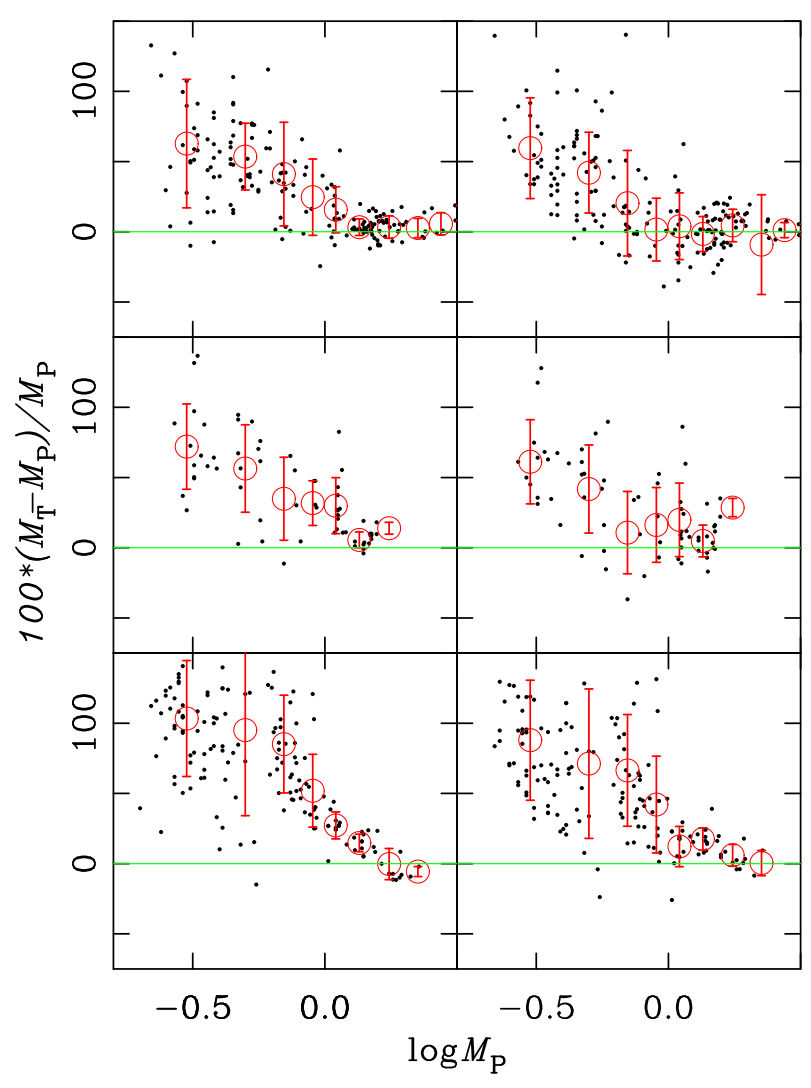

Fig. 3. Percentage difference between the masses for PMS cluster members in NGC 2264, calculated by our procedure ( $T$-values; see text) and those by D07, F06, and R02 ( $P$-values), from upper to lower panels, respectively. The quantity $100 \times\left(M_{\mathrm{T}}-M_{\mathrm{P}}\right) / M_{\mathrm{P}}$ is plotted versus $M_{\mathrm{P}}$, in the three published cases. The panels to the left show the masses calculated with respect to S00 isochrones. The panels to the right show masses calculated with respect to Y01 isochrones. Red circles and error bars show averages and rms deviations in the corresponding mass bins. The figure is to be compared to Figs. 4 and 5 in H04.

This result is indeed remarkable, since the models used in the calculation of both $T$ - and $P$-values are again the same ( $\mathrm{S} 00$ for the differences shown in the left panel of Fig. 3), and the 
photometric colours, distance and absorption for every member are also the same in both calculations. The difference for the $D 07$ values is especially striking, since the mass calculation in D07 follows a similar procedure to our $T$-procedure.

The differences shown in Fig. 3 suggest that the mass results derived from comparing observations to models in the CM diagram offer better performances than the comparison in the theoretical luminosity against effective temperature HR diagram, although these differences may change, depending on the particular calibration and model used. This is the case for different calibrations $T_{\text {eff }}+L$ from magnitudes (H04), and it also occurs when using different models or different formulas to translate the theoretical quantities to magnitudes and colours. This is exemplified in the right panels of Fig. 3, where the $T$-values calculated from comparison to Y01 isochrones are used. In this case both isochrone sets, S00 and Y01, have been transformed with the same calibration to the CM plane, and the observable differences between the two panels can therefore be ascribed to differences between the S00 and Y01 models.

\subsubsection{Mass functions}

We now discuss the results of the masses derived in our work, which can be associated with the so-called $T$-values above. The clusters are at different distances, and their photometry, for the PMS candidate members in particular, has different levels of completeness. For the present purpose, we estimate this level to be at the star whose absolute magnitude in the $5 \mathrm{Myr}$ isochrone, added to the apparent distance modulus of the cluster $(3.1 \times E(B-V)+D M)$, equals the faintest magnitude of all observed stars with photometric errors in all indices below 0.05 mag. We recall that this was a condition for considering a given star as a 3CM-PMS cluster member.

The mass functions discussed here were calculated for all member stars with a mass above the value given by this approximated completeness level. This ranges from $0.65 M_{\odot}$ for NGC 2362 and Trumpler 18, to $1.3 M_{\odot}$ for NGC 5606. The mass functions include masses estimated for MS and postMS members, obtained from the Padova isochrones (Girardi et al. 2002), also used to determine the age of the clusters (DAY07).

In Fig. 4 we plot the obtained mass functions as an example, using the SO0 models. The plot includes an indication of the Salpeter slope. In all sequences plotted in Fig. 4 one can observe the presence of a break at around $\log M \sim 0.2\left(1.5 M_{\odot}\right)$. This finding agrees with the results on NGC 1893 by Sharma et al. (2007) and the mass function obtained by Damiani et al. (2006) from independent membership estimators, although the particular values might differ. We also note the relatively flatter slopes for the clusters NGC 2362, also found by Damiani et al. (2006), and NGC 3293 (Slawson et al. 2007). The case of NGC 2362 is particularly interesting. It offers a good verification of our results, since we dispose of PMS member samples from different indicators, and mass functions have, moreover, been derived by other authors (Damiani 2006). In addition to our membership and mass estimates, we have calculated the mass function with the S00 masses for the PMS sample associated with X-ray sources (Delgado et al. 2006) and those with $\mathrm{H} \alpha$ emission and Li6707 ̊̊ absorption (Dahm 2005). The resulting mass function is plotted in Fig. 4 and shows very good agreement with the slope determined for our selected PMS sample.

In Fig. 5 we plot the slopes of the mass functions $\delta \log N_{M} / \delta \log M$ against cluster age. These are the slopes of linear least squares fits like those shown in Fig. 4, which were

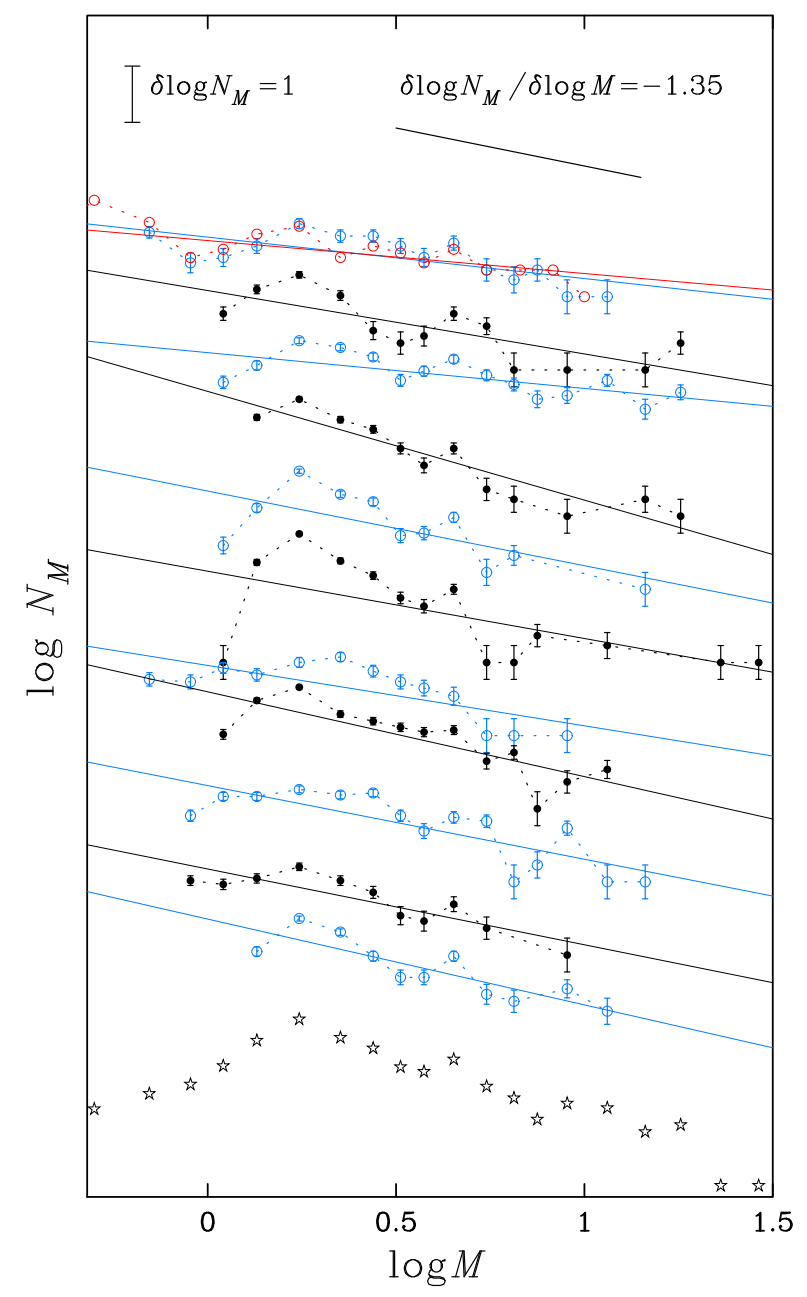

Fig. 4. Mass functions for all clusters with PMS member stars obtained from S00 models. The sequence of mass functions follows the order in Table 1 (ascending Galactic longitude). The size of the scale in the vertical axis and the Salpeter slope $(-1.35)$ are shown in the upper part of the plot. For NGC 2362, the number of members with X-ray activity, $\mathrm{H} \alpha$ emission, and Li6707 $\AA$ absorption are plotted as red circles for comparison. The joint mass function obtained by adding the member numbers of all clusters in each mass bin is plotted at the bottom as stars.

obtained for the clusters in our sample with PMS members from three models (P99, S00, and Y01). The derived mass function slopes shown in Fig. 5 show some differences between models. We observe the better agreement with the Salpeter (1955) value of the mass functions from S00 models and a slight steepening of the slope with increasing age. In general, the differences between models need assessment with independent membership determinations. Once the refined member samples have been obtained, they offer the possibility of checking the performances of the different models.

The differences in mass functions are reflected in the relative numbers of PMS members to total number of members (PMS+MS+postMS). These numbers are listed in Table 2. The PMS member number, $N_{\text {PMS }}$, and the ratio of $N_{\text {PMS }}$ to total members' number are plotted against cluster age in Fig. 6.

Several factors are present in the spread seen in this kind of plots. In addition to the general trend in the sample, the uncertainties of the plotted parameters, possible biases affecting the sample, and the real deviations due to peculiar properties of 


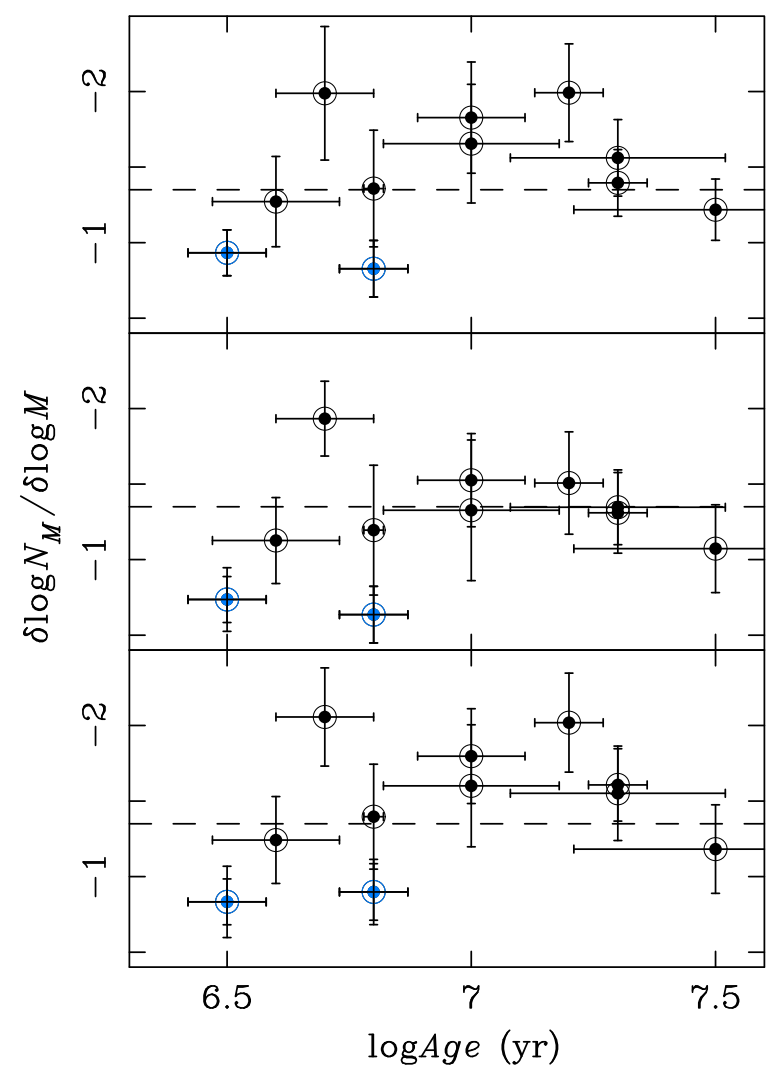

Fig. 5. Slopes of the mass functions $\left(\delta \log N_{M} / \delta \log M\right)$ for the 11 clusters in the sample as a function of cluster age. Members of mass above the approximate completeness limit defined in Sect. 3.1.2 are considered. The slopes with PMS members using three different models are plotted. From top to down: P99, S00, Y01. Vertical error bars reproduce the slope errors from the least squares fits. Horizontal broken lines mark the Salpeter IMF slope $(-1.35)$.

some objects combine to increase the spread. We consider the plot in the upper panel in Fig. 6 as an example of this. The plot can be interpreted as showing a decrease in PMS member number with cluster age for the majority of clusters, with four clusters that deviate from the trend. Three of these clusters (NGC 2367, Hogg 11, and NGC 3590), however, follow the slight decrease in the relative numbers, which can be surmised from the plot in the lower panel. This suggests that they would be different in richness of members to the other clusters in the sample, but would exhibit a "normal" relation between PMS and total member numbers. On the other hand, the location of NGC 3293 in both plots suggests that it has a relatively larger number of MS+postMS members, in agreement with the findings by Slawson et al. (2007), whereas the location of NGC 2362 in both plots would suggest a true deficit of PMS members in the cluster, a property also detected by Damiani et al. (2006). In this context, we note that NGC 2362 is located at a relatively close distance and could not fit completely in our field of view. Some PMS members could be excluded.

Vertical error bars plotted in Fig. 6 are calculated with the assumption that the number of members has an absolute error given by its square root. In spite of the uncertainties, the decreasing trend with age of the relative number in the lower plot and the deviating location of the two clusters marked can be estimated.

A final comment has to be made about the masses of the PMS candidate members. The mass functions were also calculated for

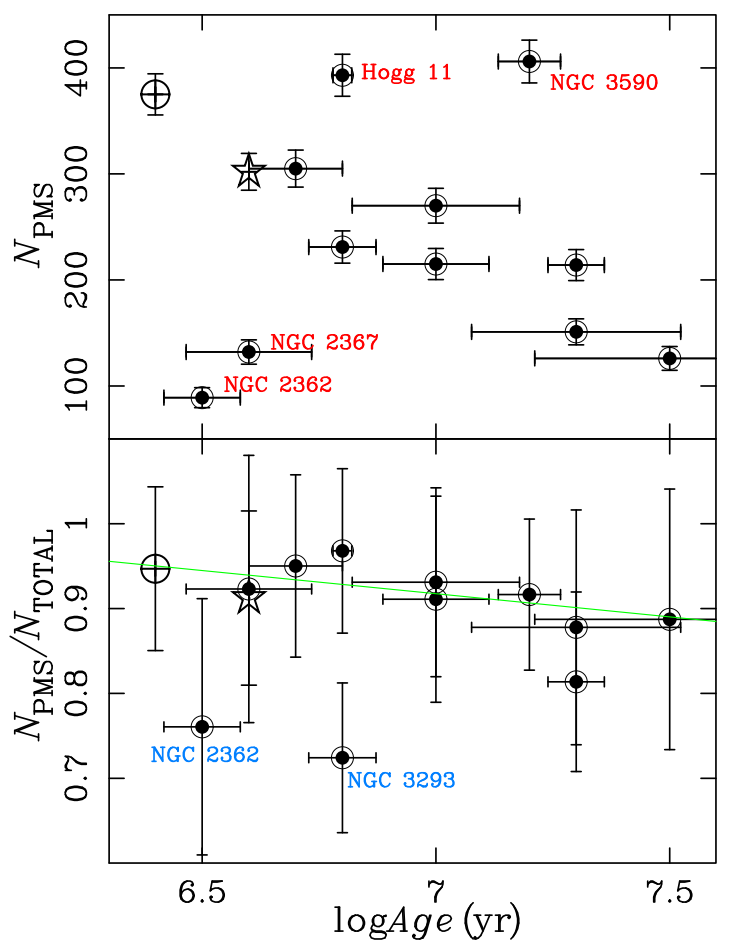

Fig. 6. Number of PMS members (upper panel) and ratio of PMS to total member numbers (PMS+MS+postMS), plotted versus cluster age. The values for NGC 2264 (crossed circle) and NGC 1893 (star) are shown for comparison. The green line in the lower plot represents a linear fit to the points, excluding the clusters marked in red with their names in the upper plot. A correlation coefficient of 0.7 is obtained. Error bars in the age are listed in Table 1. In the vertical axis, they are calculated assuming absolute error in the numbers equal to the square root of the numbers themselves.

only those PMS members with masses higher than the approximated completeness limit in each cluster defined in Sect. 3.1.2 above. In these mass ranges, we deal with AF spectral types for the selected PMS stars. For these samples, the slopes obtained are clearly steeper than the Salpeter value, also showing clear differences between the values obtained for masses from different isochrone models. In Table 2 we also list the mass function slope only for those PMS cluster members obtained with S00 models. We recall in this context the wide spread in derived mass function slopes reported by Kroupa (2002), which is particularly pronounced for young clusters, and especially in the mass range 0.8 to $2.5 M_{\odot}$. This overlaps with the lower half of the range of mass values for our selected PMS members.

\subsection{Age and spatial structure}

In this section we analyse the results of the age and spatial distributions of the PMS member sequences. As mentioned above, several factors affect the calculated parameters, with the possibly undetected peculiarities of some clusters being an important source of variation. We aim to assess the reliability of the selected member samples, which should be reflected in the presence of relations between parameters with regularities clearly beyond what could be expected from an alleatory or ineffective member selection. 


\subsubsection{Age and age spreads}

The measurement of star ages is always the result of comparison with evolutionary stellar models. This means that an assessment of models is required, and this can in turn only be achieved by comparison with the observations of the same stars whose age we want to measure. In other words, age measurement and model improvement have to be carried out in parallel, if not simultaneously.

Several methods have been proposed to improve our knowledge of PMS ages. The lithium abundance and features in the $\mathrm{CM}$ diagram are the most recently discussed ones (Naylor et al. 2009; Cignoni et al. 2010; Cargile \& James 2010). Some of them present some unsolved problems (Naylor 2009; Jeffries et al. 2009) and all methods need the input of an evolutionary model to go from relative to absolute age measurements. In addition to relative ages, one important issue in the age structure of YOCs concerns the actual presence of age spreads in the PMS member sequence, and how large this might be. The possible age spread is expected to reflect the presence of either instantaneous, continuous, or episodic star formation in the cluster (Hillenbrand 2008).

The presence of age spreads among PMS members and the age differences with the MS members in clusters is an open question. In general, age spreads in the PMS sequences are accepted, with values ranging from $2 \mathrm{Myr}$ to more than $10 \mathrm{Myr}$ (Park et al. 2002; DeGioia-Eastwood et al. 2001). Some results indicate that the formation of lower mass PMS stars continues after the most massive stars have formed, although with varying efficiency, while other studies conclude that the normal sequence of formation is in fact from lower to higher masses (Ojha et al. 2010). Some mention is made in this context to the possibility that the opposite case would result in inhibiting the formation of low-mass stars caused by radiative feedback from the more massive members (Price \& Bate 2009; Zinnecker \& Beuther 2008). The determination of age spreads in real clusters are in any case influenced by the undetected presence of binaries, which can lead to a large overestimate of the spread (Weidner et al. 2009).

With our procedure we obtain age values with uncertainties for all assigned members, as explained in Sect. 2.2. The age of the PMS sequence in each cluster is then calculated as the average of all ages for the candidate members. The rms deviation of this mean value, $\Sigma$, in principle indicates the possible age spread. The age values obtained from the four models used here (D97, P99, S00, and Y01) are included in the on-line catalogue. These values are in good agreement with previous results on different YOCs (Park \& Sung 2002; Mamajek et al. 2002). These works indicate that ages from D97 are distinctly lower than those measured using the three other models, by amounts reaching several Myr. On the other hand, differences between P99, S00, and Y01 models range at most from 0.5 to $1 \mathrm{Myr}$ but are still systematic and significant. The P99 models predict lower values than both S00 and Y01 models. These last two produce similar results. The average PMS ages of each cluster, obtained from S00 models, are listed in Table 2.

In reference to the age spreads, the use of independent membership determination from spectroscopy is especially needed to add the necessary constraints to the results from photometry and models. Without this membership confirmation, the standard deviation of the mean age, $\Sigma$, cannot be associated with a definite age spread. In spite of this shortcoming, a remarkable relation is found between the calculated standard deviation and the typical clustering scale, $\Delta$, calculated as the average value of the distances between all pairs of members. This defines the spatial

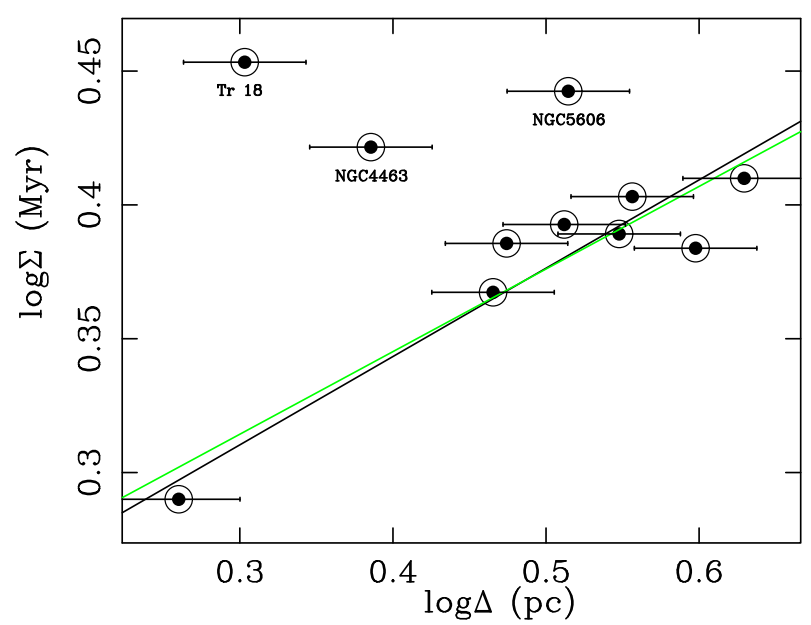

Fig. 7. Relation between the rms deviation of the men PMS age $(\Sigma)$ and the size of the clusters, given by the characteristic clustering scale $(\Delta$. See text) for PMS members of masses above the approximate completeness limit (see Sect. 3.1.2). Horizontal error bars are calculated with the assumption of a typical error in the distance modulus of 0.2 . A bootstrapping estimate of the uncertainty in $\Sigma$ gives values similar to the size of the representative points. The straight line reproduces the slope $(0.33)$ of the scaling relation between age spread and size proposed by Efremov \& Elmegreen (1998) for star-forming regions in a wide range of sizes. The green line shows a linear least squares fit, excluding the clusters marked with their names. A slope of 0.31 and a correlation coefficient of 0.8 are obtained.

concentration of the population considered (Kaas et al. 2004). In Fig. 7, $\Sigma$ is plotted against this clustering scale, which is calculated in each cluster for all pairs of PMS members with mass above the approximate completeness limit defined in Sect. 3.1.2. In the figure we overplot a line of slope 0.33 of the scaling relation between age dispersion and size proposed by Efremov \& Elmegreen (1998) for clusters and associations in a wider range of scales, both below and well above the spatial scale involved in our clusters. Three clusters do not follow the relation, probably because of undetected errors in the membership assignment. For the most outstanding case, Trumpler 18, DAY07 find signs of two clusters superimposed in the line of sight, of different ages and at well separated distances. It is one of the clusters with the largest age uncertainty in the sample. These properties probably affect the calculated spreads. Excluding the three deviating clusters, a linear fit to the data provides a slope of 0.31 , with a correlation coefficient of 0.8 . We consider that the agreement observed in Fig. 7 provides additional support to the ability of our procedure to detect PMS members in YOCs and to measure their physical properties.

\subsubsection{Spatial distribution}

The spatial distribution of cluster members is a classical matter of debate. A recent general review of this topic is contained in Sánchez \& Alfaro (2009) and centres on the distribution of PMS members in YOCs as a primary source of information on the physical properties of the star formation process, both caused from the initial parameters of the parental cloud and from the influence of various environmental conditions.

This issue has not been settled. Here we recall some related observational results. In a study of NGC 6383, Fitzgerald et al. (1978) found a ratio of 21 MS to 8 PMS members in the cluster core. Baade (1983) found a weak concentration of PMS 
members towards cluster centre in NGC 457, NGC 7380, and IC 1805, together with the presence of extended haloes, which could otherwise just be weak members, not necessarily PMS stars. In a study of IC 348, Lada \& Lada (1995) found a radial decay of both star density and subclustering, without specifying the differences between MS and PMS stars. In this same region, Herbig (1998) found a tendency of weak-lined T Tauri (WTT) stars to be more concentrated than classical T Tauri (CTT). On the other hand, he proposes a mean age of 1.4 Myr for the members inside the central 4 arcmin, where the outer population would have a mean age of $2.8 \mathrm{Myr}$. He suggests in this case that a young cluster was being observed in projection onto an older background population of PMS stars. Baume et al. (1999), regarding results on NGC 6231, propose the existence of primordial mass segregation, in the sense that lower mass stars would be located at outer locations. In their study of NGC 1893, Marco \& Negueruela (2002) find a distinct spatial distribution for MS and PMS members, not related to differences in concentration. They find a relative lack of PMS members at places where the MS members are more numerous. On the other hand, in a study of the Carina OB association, Sartori et al. (2003) find no diferences between massive MS members and PMS members, neither in the spatial distribution nor in kinematic properties or the age distribution.

The suggestions of sequential or induced star formation in star-forming regions is a topic in itself. Here we mention the results of Prisinzano et al. (2005) for NGC 6530, where they indeed find what was searched for and claimed as not present in the Carina region mentioned before: signs of spatially sequential formation, as a phenomenon different from primordial spatial segregation. The question of spatially sequential formation is currently the subject of relatively intense study, as suggested in several studies of star-forming regions (Puga et al. 2009; Delgado et al. 2010, and their references).

How then do our PMS samples reflect the properties of the spatial structure of the different clusters? The scale of spatial concentration defined in Sect. 3.2 above has been calculated for PMS stars in two separated mass ranges, $1-2 M_{\odot}$ and 2-3.5 $M_{\odot}$. These mass intervals were chosen to enhance the possible differences between spatial distributions, and were also limited to the mass range in which we are more confident, avoiding the completeness limit at the lower limit, and the merging at the upper mass limit with possible MS members.

In the upper panel of Fig. 8 we plot $\Delta$ against age for all PMS members of mass above the approximate completeness limit. Symbols for NGC 2264 and NGC 1893 are included for comparison, as in previous figures. NGC 2362 shows a relatively higher concentration for its age. A clear decreasing trend with age is observed, which can however be affected by some bias due to the distances of the different clusters. In the lower panel of Fig. 8 we plot the ratio between the concentrations of lower and higher mass PMS members (1-2 $M_{\odot}$ and 2-3.5 $\left.M_{\odot}\right)$. In spite of the large error bars, we conjecture a widening of the lower mass PMS members distribution relative to the one for PMS members of higher mass, which amounts to around $20 \%$ in the age range covered. This suggests the presence of dynamical mass segregation, in the sense of a wider distribution for lower mass stars, as the cluster age increases.

A formal relation is obtained through a linear least squares fit, also plotted in Fig. 8, with a correlation coefficient equal to 0.7 . In this fit we exclude the two most deviating points marked in the plot, which correspond to the clusters NGC 3293 and Trumpler 18. The case of Trumpler 18 has been discussed in connection with the plot in Fig. 7. The field of the cluster

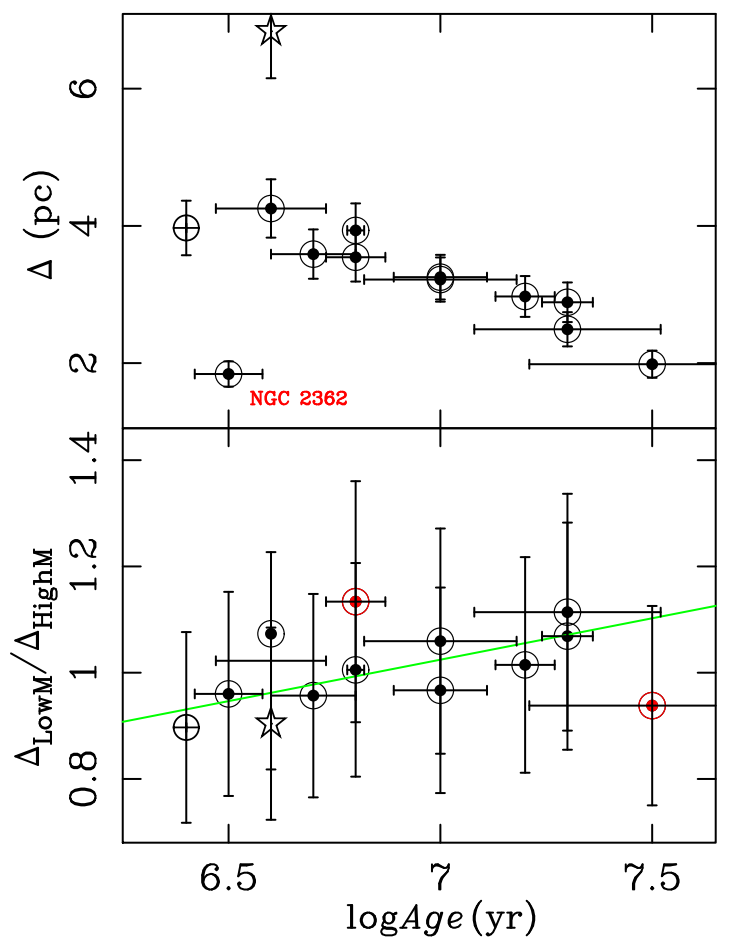

Fig. 8. Characteristic clustering scales, $\Delta$ (see text), plotted versus cluster Age. The upper panel shows $\Delta$ for PMS members of mass above the approximate completeness limit defined in Sect. 3.1.2. The relatively more concentrated NGC 2362 is marked in red. In the lower panel, the ratio between the $\Delta$ values of the lower mass (1-2 $\left.M_{\odot}\right)$ and higher mass $\left(2-3.5 M_{\odot}\right)$ PMS members is shown. Representative points for NGC 2264 and NGC 1893 are shown for comparison (crossed circle and star, respectively. See Sect. 2.3). Vertical error bars are calculated with the assumption that the member numbers have an absolute error equal to their square root. A linear squares fit with exclusion of NGC 3293 and Trumpler 18 (red symbols), and a correlation coefficient of 0.7 , is plotted as a straight green line.

is probably containing two superimposed associations, a fact which might influence the measured properties. As pointed out above, NGC 3293 has a relatively large number of massive MS members than the other clusters in the sample. Some particular features of the star formation process in the cluster could be showing up in its spatial structure.

\section{Conclusions}

We have presented the results from our procedure for establishing PMS membership in YOCs and simultaneously for measuring the physical parameters of the selected member stars. The procedure ultimately aims at checking the performances of the evolutionary models used in this task, once the third ingredient of spectroscopic observations is added to photometry and models, in order to independently confirm the membership assignments.

At present, the status of the procedure already appears to be not only promising but actually able to provide valuable results. This discussion has led us to suggest some relations between the parameters measured, which agree with previous findings. Independently of these relations, affected by different sources of uncertainty, the main result consists in finding regularities that could not be plausibly expected if the member selections were not well founded. In the present paper we refer to 11 clusters in 
an age range between 4 and $30 \mathrm{Myr}$, as determined from comparing the upper main sequence to isochrones in the CM diagrams.

1. Using different procedures but the same published photometry, we found different results for the masses of NGC 2264 PMS members. This suggests that evaluating stellar masses from a comparison with isochrones in the CM diagrams leads to mass values that are in better agreement with dynamical masses than those when they are evaluated from a comparison with isochrones in the theoretical HR diagram.

2. The derived mass functions for the PMS selected member sequences in general show slopes that agree with the Salpeter value when using the S00 models, while steeper slopes are obtained when using P99 and Y01 models. A slight increase in the slope with increasing cluster age is suggested. The slopes found for NGC 3293 and NGC 2362 are flatter than the Salpeter value. In the case of NGC 2362, for which independent membership assignments are available, this finding coincides with other estimates of the mass function.

3. The flatter slopes found for the mass functions of NGC 2362 and NGC 3293 are reflected in a particularly low ratio between the number of PMS members and the total members number. Whereas NGC 3293 contains a larger number of massive MS members than the trend shown by the other clusters, for NGC 2362 this lower ratio probably originates in a deficit of lower mass PMS members.

4. Obtaining PMS ages with the models used reproduces findings in previous studies, in the sense of increasingly predicted ages from models in the order D97, P99, S00. The ages from the Y01 model are indistinguishable from those in the S00 models, whereas the D97 models predict distinctly lower ages, which amount to several Myr less than the other models.

5. The standard deviation in the mean PMS age is in the range 2 to $3 \mathrm{Myr}$ in all clusters. This agrees with general findings on age spread values claimed in several studies of YOCs, but it really needs the assessment of the membership assignments to be confirmed. However, the plot of this quantity versus cluster size, measured as the average of distances between all pairs of PMS members, shows interesting agreement with previously found scaling relations between age spread and size for star-forming regions across a wide range of scales.

6. The ratio between characteristic clustering scales for PMS members in the mass ranges $1-2 M_{\odot}$ and 2-3.5 $M_{\odot}$ shows a marginal positive correlation with cluster age. This would suggest the presence of increasing dynamical mass segregation with increasing cluster age, in the sense that lower mass PMS members show a relatively wider distribution for older clusters. Although marginal, we consider this as additional support for the procedure of PMS members selection.

To sum up, CCD wide band photometry can be obtained for practically all young clusters known in the Galactic disk with the use of small to medium size telescopes. For most of them, precise photometry can be obtained for expected PMS cluster members down to spectral type K0, and even fainter, depending on distance and absorption. Is a consistent wealth of information on PMS evolution and physics achievable with these observations? We think that the results in this study show that indeed there is. With our procedure, we simultaneously assign cluster membership from multiband cluster photometry and measure the mass and age of the individual member candidates. Spectroscopic observations, as a third foot of the tripod in addition to photometry and models, are needed to achieve a stable set of results. However, the joint use of photometry and models discussed here is already shown to be a promising procedure for the investigation of PMS stars in YOCs.

Acknowledgements. This work has been supported by the Spanish Ministerio de Educación y Ciencia through grant AYA2007-64052 and by the Consejera de Educacin y Ciencia de la Junta de Andaluca through TIC101. We made use of the NASA ADS Abstract Service and of the WEBDA data base, developed by Jean-Claude Mermilliod at the Laboratory of Astrophysics of the EPFL (Switzerland), and further developed and maintained by Ernst Paunzen at the Institute of Astronomy of the University of Vienna (Austria). This publication makes use of data products from the Two Micron All Sky Survey, which is a joint project of the University of Massachusetts and the Infrared Processing and Analysis Center/California Institute of Technology, funded by the National Aeronautics and Space Administration and the National Science Foundation.

\section{References}

Baade, D. 1983, A\&AS, 51, 235

Baume, G., Vázquez, R. A., \& Feinstein, A. 1999, A\&AS, 137, 233

Baxter, E. J., Covey, K. R., Muench, A. A., et al. 2009, AJ, 138, 963

Beccari, G., Spezzi, L., De Marchi, G., et al. 2010, ApJ, 720, 1108

Caramazza, M., Micela, G., Prisinzano, L., et al. 2008, A\&A, 488, 211

Cignoni, M., Tosi, M., Sabbi, E., et al. 2010, ApJ, 712, L63

Cargile, P. A., \& James, D. J. 2010, AJ, 140, 677

Dahm, S. E. 2005, AJ, 130, 1805

Dahm, S. E. 2008, Handbook of Star Forming Regions, ed. Bo Reipurth, The Northern Sky ASP Monograph Publications, Vol. I, 4, 966

Dahm, S. E., Simon, T., Proszkow, E. M., et al. 2007, AJ, 134, 999 (D07)

Damiani, F., Micela, G., Sciortino, S., et al. 2006, A\&A, 460, 133

D'Antona, F., \& Mazzitelli, I. 1997, MemSAIt, 68, 807 (D97)

DeGioia-Eastwood, K., Throop, H., Walker, G., et al. 2001, ApJ, 549, 578

Delgado, A. J., Alfaro, E. J., Moitinho, A., et al. 1998, AJ, 116, 1801

Delgado, A. J., Alfaro, E. J., \& Yun, J. L. 2007, A\&A, 467, 1397 (DAY07)

Efremov, Y. N., \& Elmegreen, B. G. 1998, MNRAS, 299, 588

Fitzpatrick, E. L. 1999, PASP, 111, 63

Fitzgerald, M. P., Jackson, P. D., Luiken, M., et al. 1978, MNRAS, 182, 607

Flaccomio, E., Micela, G., \& Sciortino, S. 2006, A\&A, 455, 903 (F06)

Girardi, L., Bertelli, G., Bressan, A., et al. 2002, A\&A, 391, 195

Haisch, K. E., Jr., Lada, E. A., \& Lada, C. J. 2001, ApJ, 553, L153

Herbig, G. H. 1998, ApJ, 497, 736

Hillenbrand, L. A., \& White, R. J. 2004, ApJ, 604, 741 (H04)

Hillenbrand, L. A., Bauermeister, A., \& White, R. J. 2008, ASPC, 384, 200

Jeffries, R. D., Jackson, R. J., James, D. J., et al. 2009, MNRAS, 400, 317

Kenyon, S. J., \& Hartmann, L. 1995, ApJS, 101, 117

Lada, E. A., \& Lada, C. J. 1995, AJ, 109, 1682

Mamajek, E. E., Meyer, M. R., \& Liebert, J. 2002, AJ, 124, 1670

Marco, A., \& Negueruela, I. 2002, A\&A, 393, 195

Naylor, T. 2009, MNRAS, 399, 432

Naylor, T., Mayne, N. J., Jeffries, R. D., et al. 2009, The Ages of Stars, ed. E. E. Mamajek, D. R. Soderblom, \& R. F. G. Wyse (New York: Cambridge University Press), IAU Symp., 258, 103

Ojha, D. K., Kumar, M. S. N., Davis, C. J., et al. 2010, MNRAS, 407, 1807

Palla, F., \& Stahler, S. W. 1999, ApJ, 525, 772 (P99)

Park, B.-G., \& Sung, H. 2002, AJ, 123, 892

Park, B.-G., Sung, H., Bessell, M. S., et al. 2000, AJ, 120, 894

Price, D. J., \& Bate, M. R. 2009, MNRAS, 398, 33

Prisinzano, L., Damiani, F., Micela, G., et al. 2005, A\&A, 430, 941

Rebull, L. M., Hillenbrand, L. A., Strom, S. E., et al. 2000, AJ, 119, 3026

Rebull, L. M., Makidon, R. B., Strom, S. E., et al. 2002, AJ, 123, 1528 (R02)

Salpeter, E. E. 1955, ApJ, 121, 161

Sánchez, N., \& Alfaro, E. J. 2009, ApJ, 696, 2086

Sartori, M. J., Lépine, J. R. D., \& Dias, W. S. 2003, A\&A, 404, 913

Sharma, S., Pandey, A. K., Ojha, D. K., et al. 2007, MNRAS, 380, 1141

Sicilia-Aguilar, A., Hartmann, L. W., Hernández, J., et al. 2005, AJ, 130, 188

Siess, L., Dufour, E., \& Forestini, M. 2000, A\&A, 358, 593 (S00)

Slawson, R. W., Ninkov, Z., \& Elliott, P. 2007, Ap\&SS, 312, 171

Sung, H., Bessell, M. S., \& Chun, M.-Y. 2004, AJ, 128, 1684

Walker, M. F. 1956, ApJS, 2, 365

Weidner, C., Kroupa, P., \& Maschberger, T. 2009, MNRAS, 393, 663

Yi, S., Demarque, P., Kim, Y.-Ch., et al. 2001, ApJS, 136, 417 (Y01)

Zwintz, K., Guenther, D., \& Kallinger, T. 2008, CoAst, 157, 256

Zinnecker, H., \& Beuther, H. 2008, ASPC, 387, 438 